\title{
COMBINATORIAL PROBLEMS IN FINITE FIELDS AND SIDON SETS
}

\author{
JAVIER CILLERUELO
}

\begin{abstract}
We use Sidon sets to present an elementary method to study some combinatorial problems in finite fields, such as sum product estimates, solubility of some equations and distribution of sequences in small intervals. We obtain classic and more recent results avoiding the use of exponential sums, the usual tool to deal with these problems.
\end{abstract}

\section{INTRODUCTION}

The aim of the present work is to introduce a new elementary method to study a class of combinatorial problems in finite fields: incidence problems, sumproduct estimates, solubility of some equations, distribution of sequences in small intervals, etc.

The main tool in our approach are Sidon sets, which are important objects in combinatorial number theory.

In Section $\S 2$ we present Theorem 2.1, which is a new result about Sidon sets and the main tool in our method. To illustrate how this method works, we include in this section two easy application of this theorem. The first one recovers a result of Vinh [15] about the number of incidences between $P$ points and $L$ lines in a field $\mathbb{F}_{q}$. The second one proves that if $A=\left\{g^{x}: 0 \leq x \leq(\sqrt{2}+o(1)) p^{3 / 4}\right\}$ and $g$ is a primitive root modulo $p$, then $A-A=\mathbb{Z}_{p}$. This improves previous results obtained by Garaev-Kueh [4], Konyagin [9] and García [6].

Section $\S 3$ is devoted to sum-product estimates. Garaev [2] used character sums to give the nontrivial lower estimate $\max (|A+A|,|A A|) \gg \min \left(\sqrt{|A| q},|A|^{2} / \sqrt{q}\right)$. Theorem [3.1, which is an easy consequence of Theorem 2.1, gives a nontrivial upper bound for the number of elements of a dense Sidon set in an arbitrary set $B$ when $|B+B|$ is small. We use this upper bound to give a quick proof of Garaev's estimate and related results.

Sárközy [11, 12] proved the solubility of the equations $x_{1} x_{2}+x_{3} x_{4}=1$ and $x_{1} x_{2}=x_{3}+x_{4}, x_{i} \in A_{i}$ for arbitrary sets $A_{i} \subset \mathbb{F}_{p}$ when $\left|A_{1}\right|\left|A_{2}\right|\left|A_{3}\right|\left|A_{4}\right| \gg p^{3}$. This result was extended to any field $\mathbb{F}_{q}$ in [7]. The proof is based in estimates of exponential sums and they asked for an elementary algebraic proof of the solubility of these equations (problem 3 of [1]). Our method provides a proof 
of this kind. Actually, Sarkőzy's equations are special cases of more general equations which we study in section $\S 4$.

In section $\S 5$, we apply our method to study the distribution of some sequences in $\mathbb{Z}_{p}$. As an example we prove that if $g$ is a primitive root modulo $p$, then for any intervals $I, J$ and any positive integer $r$ we have

$$
\left|\left\{(x, y) \in I \times J: g^{x}-g^{y} \equiv 1 \quad(\bmod p)\right\}\right|=\frac{|I||J|}{p}+\theta_{r}\left(\left(\frac{|I||J|}{p^{3 / 2}}\right)^{1 / r}+1\right) \sqrt{p},
$$

with $\left|\theta_{r}\right| \leq 4^{r}$. The error term is smaller than the error term obtained by Garaev [3].

\section{Sidon SETS}

Let $G$ be a finite abelian group. For any sets $A, B \subset G$ and $x \in G$, we write $r_{A-B}(x)$ for the number of representations of $x=a-b, a \in A, b \in B$. We have the well known identities

$$
\begin{aligned}
& \sum_{x \in G} r_{A-B}(x)=|A||B| \\
& \sum_{x \in G} r_{A-B}^{2}(x)=\sum_{x \in G} r_{A-A}(x) r_{B-B}(x) .
\end{aligned}
$$

Definition 1. We say that a set $\mathcal{A} \subset G$ is a Sidon set if $r_{\mathcal{A}-\mathcal{A}}(x) \leq 1$ whenever $x \neq 0$.

By counting the number of differences $a-a^{\prime}$, we can see that if $\mathcal{A}$ is a Sidon set, then $|\mathcal{A}|<\sqrt{|G|}+1 / 2$. The most interesting Sidon sets are those which have large cardinality, that is, $|\mathcal{A}|=\sqrt{|G|}-\delta$ where $\delta$ is a small number. We state our main theorem.

Theorem 2.1. Let $\mathcal{A}$ be a Sidon set in a finite abelian group $G$ with $|\mathcal{A}|=$ $\sqrt{|G|}-\delta$. Then, for all $B, B^{\prime} \subset G$ we have

$$
\left|\left\{\left(b, b^{\prime}\right) \in B \times B^{\prime}, b+b^{\prime} \in \mathcal{A}\right\}\right|=\frac{|\mathcal{A}|}{|G|}|B|\left|B^{\prime}\right|+\theta\left(|B|\left|B^{\prime}\right|\right)^{1 / 2}|G|^{1 / 4},
$$

with $|\theta|<1+\frac{|B|}{|G|} \max (0, \delta)$.

Proof. Since $\mathcal{A}$ is a Sidon set,

$$
\begin{aligned}
\sum_{x \in G} r_{B-B}(x) r_{\mathcal{A}-\mathcal{A}}(x) & =|\mathcal{A}||B|+\sum_{x \neq 0} r_{B-B}(x) r_{\mathcal{A}-\mathcal{A}}(x) \\
& \leq|\mathcal{A}||B|+\sum_{x \neq 0} r_{B-B}(x)=|\mathcal{A}||B|+|B|^{2}-|B|
\end{aligned}
$$


Using this inequality and identities (2.1) and (2.2) we have

$$
\begin{aligned}
\sum_{x \in G}\left(r_{\mathcal{A}-B}(x)-\frac{|\mathcal{A}||B|}{|G|}\right)^{2} & =\sum_{x \in G} r_{B-B}(x) r_{\mathcal{A}-\mathcal{A}}(x)-\frac{|\mathcal{A}|^{2}|B|^{2}}{|G|} \\
& \leq|B|(|\mathcal{A}|-1)+|B|^{2} \frac{|G|-|\mathcal{A}|^{2}}{|G|}
\end{aligned}
$$

We observe that

$$
\left|\left\{\left(b, b^{\prime}\right) \in B \times B^{\prime}, b+b^{\prime} \in \mathcal{A}\right\}\right|-\frac{|B|\left|B^{\prime}\right||\mathcal{A}|}{|G|}=\sum_{b^{\prime} \in B^{\prime}}\left(r_{\mathcal{A}-B}\left(b^{\prime}\right)-\frac{|\mathcal{A}||B|}{|G|}\right) .
$$

Applying the Cauchy-Schwarz inequality, taking $(2.3)$ and $|\mathcal{A}|=|G|^{1 / 2}-\delta$ into account we obtain

$$
\begin{aligned}
\left|\sum_{b^{\prime} \in B^{\prime}}\left(r_{\mathcal{A}-B}\left(b^{\prime}\right)-\frac{|\mathcal{A}||B|}{|G|}\right)\right|^{2} & \leq\left|B^{\prime}\right|\left(|B|(|\mathcal{A}|-1)+|B|^{2} \frac{|G|-|\mathcal{A}|^{2}}{|G|}\right) \\
& =\left|B^{\prime}\right||B|\left(|G|^{1 / 2}-\delta-1+|B| \frac{\delta\left(2|G|^{1 / 2}-\delta\right)}{|G|}\right) \\
& <|B|\left|B^{\prime}\right||G|^{1 / 2}\left(1+2 \max (0, \delta) \frac{|B|}{|G|}\right) .
\end{aligned}
$$

The Sidon sets we will consider in applications satisfy $\delta \leq 1$ and $|B|=o(|G|)$. In these cases we have $|\theta| \leq 1+o(1)$.

2.1. Examples of dense Sidon sets. The three families of Sidon sets we will describe next, have maximal cardinality in their ambient group $G$. Let $g$ be a generator of $\mathbb{F}_{q}^{*}$.

Example 1. Let $p(x), r(x) \in \mathbb{F}_{q}[X]$ be polynomials of degree $\leq 2$ such that $p(x)-$ $\mu r(x)$ is not a constant for any $\mu \in \mathbb{F}_{q}$. The set

$$
\mathcal{A}=\left\{(p(x), r(x)): x \in \mathbb{F}_{q}\right\}
$$

is a Sidon set in $\mathbb{F}_{q} \times \mathbb{F}_{q}$. In particular, the set $\mathcal{A}=\left\{\left(x, x^{2}\right): x \in \mathbb{F}_{q}\right\}$ is a Sidon set.

We have to check that when $\left(e_{1}, e_{2}\right) \neq(0,0)$ the relation $\left(p\left(x_{1}\right), r\left(x_{1}\right)\right)-$ $\left(p\left(x_{2}\right), r\left(x_{2}\right)\right)=\left(e_{1}, e_{2}\right)$ uniquely determines $x_{1}$ and $x_{2}$. If $p(x)$ is linear then from $p\left(x_{1}\right)-p\left(x_{2}\right)=e_{1}$ we obtain $x_{1}=x_{2}+\lambda$ for some $\lambda$. Thus, $r\left(x_{2}+\lambda\right)-r\left(x_{2}\right)=e_{2}$ is a linear equation and we obtain $x_{2}$ and then $x_{1}$. If $p(x)$ is quadratic we consider $\mu$ such that $p(x)-\mu r(x)$ is a linear polynomial and we proceed as above. 
Example 2. For any generator $g$ of $\mathbb{F}_{q}^{*}$, the set

$$
\mathcal{A}=\left\{\left(x, g^{x}\right): x \in \mathbb{Z}_{q-1}\right\}
$$

is a Sidon set in $\mathbb{Z}_{q-1} \times \mathbb{F}_{q}$.

Sometimes we will describe this set as $\mathcal{A}=\left\{(\log x, x): x \in \mathbb{F}_{q}^{*}\right\}$ where $\log x=$ $\log _{g} x$ is the discrete logarithm.

From $\left(x_{1}, g^{x_{1}}\right)-\left(x_{2}, g^{x_{2}}\right)=\left(e_{1}, e_{2}\right) \neq(0,0)$ we have $x_{1}-x_{2} \equiv e_{1}(\bmod q-1)$ and hence $g^{x_{1}}=g^{e_{1}+x_{2}}$. Putting this in $g^{x_{1}}-g^{x_{2}}=e_{2}$ we get $g^{x_{2}}\left(g^{e_{1}}-1\right)=e_{2}$.

If $e_{1}=0$ then $e_{2}=0$, but we have assumed that $\left(e_{1}, e_{2}\right) \neq(0,0)$. If $e_{1} \neq 0$ the last equality determines $x_{2}$, and then $x_{1}$.

Example 3. For any pair of generators $g_{1}, g_{2}$ of $\mathbb{F}_{q}^{*}$, the set

$$
\mathcal{A}=\left\{(x, y) \in \mathbb{Z}_{q-1} \times \mathbb{Z}_{q-1}: g_{1}^{x}+g_{2}^{y}=1\right\}
$$

is a Sidon set in $\mathbb{Z}_{q-1} \times \mathbb{Z}_{q-1}$. Since translations preserve Sidoness property, for any $\lambda \neq 0$, the sets $\mathcal{A}=\left\{(x, y): g_{1}^{x}+g_{2}^{y}=\lambda\right\}$ and $\mathcal{A}=\left\{(x, y): g_{1}^{x}-g_{2}^{y}=\lambda\right\}$ are also Sidon sets.

To see that $\mathcal{A}$ is a Sidon set we have to prove that if $\left(e_{1}, e_{2}\right) \neq(0,0)$ then the equation $\left(x_{1}, y_{1}\right)-\left(x_{2}, y_{2}\right)=\left(e_{1}, e_{2}\right)$ determines $x_{1}, x_{2}$ under the conditions $g_{1}^{x_{1}}+g_{2}^{y_{1}}=g_{1}^{x_{2}}+g_{2}^{y_{2}}=1$ in $\mathbb{F}_{q}$. We observe that $x_{1}-x_{2} \equiv e_{1}(\bmod (q-1))$ and $y_{1}-y_{2} \equiv e_{2}(\bmod (q-1))$ imply that $g_{1}^{x_{1}}=g_{1}^{x_{2}+e_{1}}$ and $g_{2}^{y_{1}}=g_{2}^{y_{2}+e_{2}}$ in $\mathbb{F}_{q}$ and we obtain $g_{1}^{x_{2}+e_{1}}+g_{2}^{y_{2}+e_{2}}=g_{1}^{x_{2}}+g_{2}^{y_{2}}=1$ in $\mathbb{F}_{q}$. Thus $g_{2}^{y_{2}}\left(g_{2}^{e_{2}}-g_{1}^{e_{1}}\right)=1-g_{1}^{e_{1}}$. If $e_{1} \neq 0$ and $g_{2}^{e_{2}} \neq g_{1}^{e_{1}}$ we obtain $y_{2}$ and then $x_{2}, x_{1}$ and $y_{1}$. If $e_{1}=0$ or $g_{2}^{e_{2}}=g_{1}^{e_{1}}$, the equation has not solutions unless $\left(e_{1}, e_{2}\right)=(0,0)$.

When $q=p$ is a prime number we can identify $\mathbb{F}_{p}$ with $\mathbb{Z}_{p}$. We ilustrate in the pictures below the three examples of Sidon sets described above.

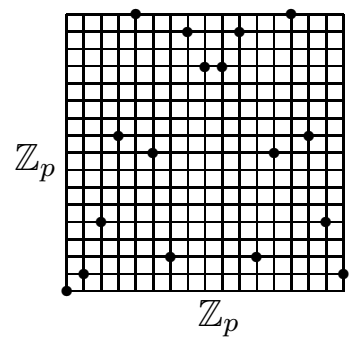

Example 1

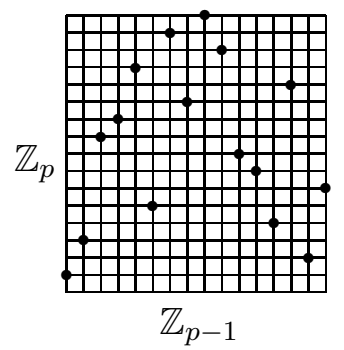

Example 2

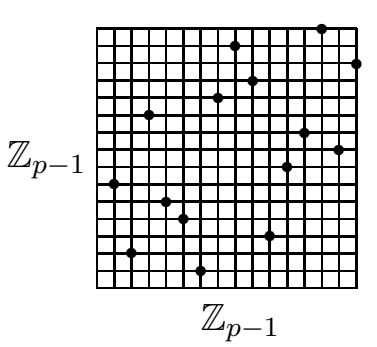

Example 3

The Sidon sets given in examples, with $q, q-1$ and $q-2$ elements respectively, have maximal cardinality in their ambient groups. The values of $\delta=|G|^{1 / 2}-|\mathcal{A}|$ are $\delta=0,1 / 2-o(1)$ and 1 respectively. We finish this section with two easy applications of Theorem 2.1 . 
2.2. Incidence of lines and points in $\mathbb{F}_{q} \times \mathbb{F}_{q}$. Let $I(P, L)=\mid\{(p, l) \in P \times L$ : $p \in L\} \mid$ be the number of incidences between a set $P$ of points and set $L$ of lines in $\mathbb{F}_{q} \times \mathbb{F}_{q}$, that is

$$
I(P, L)=|\{(p, l) \in P \times L: p \in l\}| .
$$

Vinh [15] proved that $I(P, L) \leq \frac{|P||L|}{p}+O(\sqrt{|P||L| p})$. We recover this result as a straightforward consequence of Theorem 2.1.

Theorem 2.2. Let $L$ be a set of lines and let $P$ be a set of points in $\mathbb{F}_{q} \times \mathbb{F}_{q}$. The following asymptotic formula holds:

$$
I(P, L)=\frac{|P||L|}{q}+O(\sqrt{|P||L| q}) .
$$

Proof. Let $L=\left\{y=\lambda_{i} x+\mu_{i}: 1 \leq i \leq|L|\right\}$ and $P=\left\{\left(p_{j}, q_{j}\right): 1 \leq j \leq|P|\right\}$. We consider the set $\mathcal{A}=\{(\log x, x)\}$ described in (2) and the sets

$$
B=\left\{\left(\log \lambda_{i},-\mu_{i}\right): 1 \leq i \leq|L|\right\}, \quad B^{\prime}=\left\{\left(\log p_{j}, q_{j}\right): 1 \leq j \leq|P|\right\} .
$$

We observe that each incidence corresponds to a solution of $\lambda_{i} p_{j}=q_{j}-\mu_{i}$ and the number of solutions of this equation is $\left|\left\{\left(b, b^{\prime}\right) \in B \times B^{\prime}: b+b^{\prime} \in \mathcal{A}\right\}\right|$. The result follows in view of Theorem 2.1.

2.3. The difference set $\left\{g^{x}-g^{y}: 0, \leq x, y \leq L\right\}$. Let $g$ be a primite root modulo $p$. Many authors have studied the problem of determining the smallest number $M$ such that $\left\{g^{x}-g^{y}: 0 \leq x, y \leq M\right\}=\mathbb{Z}_{p}$.

From the result of Rudnick and Zaharescu [10] it follows that one can take any integer $M \geq c_{0} p^{3 / 4} \log p$ where $c_{0}$ is a suitable constant. This range has been improved to $M>c p^{3 / 4}$ by Garaev and Kueh [4] and independently by Konyagin [9]. The best known admissible value for the constant $c$ has been $c=2^{5 / 4}$ due to García [6]. Our approach improves this further to the following statement.

Theorem 2.3. Let $g$ be a generator of $\mathbb{F}_{p}^{*}$. For any $\epsilon>0$ and $p>p(\epsilon)$ we have

$$
\left\{g^{x}-g^{y}: 0 \leq x, y<(\sqrt{2}+\epsilon) p^{3 / 4}\right\}=\mathbb{F}_{p} .
$$

Proof. Suppose $\lambda \notin\left\{g^{x}-g^{y}: 0 \leq x, y \leq L\right\}$ and consider in $G=\mathbb{Z}_{p-1} \times \mathbb{Z}_{p-1}$ the Sidon set $\mathcal{A}=\left\{(x, y): g^{x}-g^{y}=\lambda\right\}$. We observe that

$$
(x, y) \in \mathcal{A} \Longleftrightarrow(y, x)+\left(\frac{p-1}{2}, \frac{p-1}{2}\right) \in \mathcal{A} .
$$

Thus, it is clear that if $b, b^{\prime} \in B=[0, L / 2]^{2}+\left\{(0,0),\left(\frac{p-1}{2}, \frac{p-1}{2}\right)\right\}$ then $b+b^{\prime} \notin \mathcal{A}$. In other words, $\left|\left\{\left(b, b^{\prime}\right) \in B \times B, b+b^{\prime} \in \mathcal{A}\right\}\right|=0$.

On the other hand, Theorem 2.1 implies that

$$
0=\left|\left\{\left(b, b^{\prime}\right) \in B \times B, b+b^{\prime} \in \mathcal{A}\right\}\right| \geq \frac{|\mathcal{A}||B|^{2}}{|G|}-\left(1+\frac{|B|}{|G|}\right)|B||G|^{1 / 4} .
$$


Thus

$$
|B| \leq \frac{|G|^{5 / 4}}{|\mathcal{A}|-|G|^{1 / 4}}=\frac{(p-1)^{5 / 2}}{p-2-(p-1)^{1 / 2}}<p^{3 / 2}(1+o(1))
$$

and the theorem follows in view of $|B|=2(1+[L / 2])^{2}$.

\section{Sum-Product estimates}

We will deduce some sum-product estimates form the following lemma.

Lemma 3.1. Let $\mathcal{A}$ be a Sidon set in $G$ with $|\mathcal{A}|=|G|^{1 / 2}-\delta$. For any subsets $B, B^{\prime} \subset G$ we have

$$
|\mathcal{A} \cap B| \leq \frac{\left|B+B^{\prime}\right||\mathcal{A}|}{|G|}+\theta\left(\frac{\left|B+B^{\prime}\right|}{\left|B^{\prime}\right|}\right)^{1 / 2}|G|^{1 / 4},
$$

for some $\theta$ with $|\theta| \leq 1+\max (0, \delta) \frac{\left|B^{\prime}\right|}{|G|}$.

Proof. Indeed, by Theorem 2.1,

$$
\begin{aligned}
\left|B^{\prime}\right||\mathcal{A} \cap B| & =\left|\left\{\left(-b^{\prime}, b+b^{\prime}\right): b \in B, \quad b^{\prime} \in B^{\prime},-b^{\prime}+\left(b+b^{\prime}\right) \in \mathcal{A}\right\}\right| \\
& \leq\left\{\left(b^{\prime}, b^{\prime \prime}\right): b^{\prime} \in\left(-B^{\prime}\right) \times\left(B+B^{\prime}\right), \quad b^{\prime}+b^{\prime \prime} \in \mathcal{A}\right\} \mid \\
& \leq \frac{|\mathcal{A}|\left|B^{\prime}\right|\left|B+B^{\prime}\right|}{|G|}+\theta \sqrt{\left|B^{\prime}\right|\left|B+B^{\prime}\right|}|G|^{1 / 4}
\end{aligned}
$$

where $|\theta| \leq 1+\max (0, \delta) \frac{\left|B^{\prime}\right|}{|G|}$. The lemma follows.

Theorem 3.1 (Garaev [2]). Let $A_{1}, A_{2} \subset \mathbb{F}_{q}^{*}$ and $A_{3} \subset \mathbb{F}_{q}$. We have

$$
\max \left(\left|A_{1} A_{2}\right|,\left|A_{1}+A_{3}\right|\right) \gg \min \left(\sqrt{\left|A_{1}\right| q}, \sqrt{\left|A_{1}\right|^{2}\left|A_{2}\right|\left|A_{3}\right| / q}\right) .
$$

Proof. We consider the Sidon set $\mathcal{A}=\left\{(\log x, x): x \in \mathbb{F}_{q}^{*}\right\}$ described in example 2 and the sets $B=\left(\log A_{1}\right) \times A_{1}$ and $B^{\prime}=\left(\log A_{2}\right) \times A_{3}$. Since all the elements $\left(\log a_{1}, a_{1}\right)$ are in $\mathcal{A}$ we have that $|\mathcal{A} \cap B|=\left|A_{1}\right|$. On the other hand we observe that $\left|B+B^{\prime}\right|=\left|A_{1} A_{2}\right|\left|A_{1}+A_{3}\right|$. Lemma 3.1 implies the inequality

$$
\left|A_{1}\right| \leq \frac{\left|A_{1} A_{2}\right|\left|A_{1}+A_{3}\right|}{q}+\theta \sqrt{q \frac{\left|A_{1} A_{1}\right|\left|A_{1}+A_{3}\right|}{\left|A_{2}\right|\left|A_{3}\right|}}, \quad|\theta| \leq 1,
$$

which in turn implies (3.1).

We can mimic this proof to get the following sum-product estimates.

Theorem 3.2 (Garaev-Shen [5]). Let $A_{1}, A_{2}, A_{3} \subset \mathbb{F}_{q}^{*}$. We have

$$
\max \left(\left|\left(A_{1}+1\right) A_{2}\right|,\left|A_{1} A_{3}\right|\right) \gg \min \left(\sqrt{\left|A_{1}\right| q}, \sqrt{\left|A_{1}\right|^{2}\left|A_{2}\right|\left|A_{3}\right| / q}\right) .
$$


Proof. We consider the Sidon set $\mathcal{A}=\left\{(x, y): g^{x}-g^{y}=1\right\}$, the sets $B=$ $\log \left(A_{1}+1\right) \times \log A_{1}$ and $B^{\prime}=\log A_{2} \times \log A_{3}$ and proceed as in the proof of Theorem 3.1.

Theorem 3.3 (Solymosi [14], Hart-Li-Shen [8]). Let $p(x), q(x) \in \mathbb{F}_{q}[X]$ be polynomials of degree $\leq 2$ such that $p(x)-\mu q(x)$ is not a constant for any $\mu \in \mathbb{F}_{q}$. For any $A_{1}, A_{2}, A_{3} \subset \mathbb{F}_{q}$ we have

$$
\max \left(\left|p\left(A_{1}\right)+A_{2}\right|,\left|q\left(A_{1}\right)+A_{3}\right|\right) \gg \min \left(\sqrt{\left|A_{1}\right| q}, \sqrt{\left|A_{1}\right|^{2}\left|A_{2}\right|\left|A_{3}\right| / q}\right) .
$$

Proof. We consider the Sidon set $\mathcal{A}=\left\{(p(x), q(x)): x \in \mathbb{F}_{q}\right\}$, the sets $B=$ $p\left(A_{1}\right) \times q\left(A_{1}\right)$ and $B^{\prime}=A_{2} \times A_{3}$ and proceed as in the proof of Theorem 3.1 .

Solymosi [14] proved that if $\left\{(x, f(x)): x \in \mathbb{F}_{q}\right\} \subset \mathbb{F}_{q} \times \mathbb{F}_{q}$ is a Sidon set then $\max (|A+A|,|f(A)+f(A)|) \gg \min \left(\sqrt{|A| q},|A|^{2} / \sqrt{q}\right)$.

\section{Equations in $\mathbb{F}_{q}$}

We start with the easiest example which, however, we have not seen in the literature.

Theorem 4.1. For any $x \in \mathbb{F}_{q}$, let $X(x), Y(x)$ be any pair of subsets of $\mathbb{F}_{q}$ and put $T=\left(\sum_{x}|X(x)|\right)\left(\sum_{x}|Y(x)|\right)$. Then, the number of solutions $S$ of

$$
x^{\prime}+y^{\prime}=(x+y)^{2}, \quad x^{\prime} \in X(x), y^{\prime} \in Y(y)
$$

is

$$
S=\frac{T}{q}+\theta \sqrt{q T}
$$

for some $\theta$ with $|\theta| \leq 1$.

Proof. We consider the Sidon set $\mathcal{A}=\left\{\left(x, x^{2}\right): x \in \mathbb{F}_{q}\right\}$ and the sets

$$
B=\left\{\left(x, x^{\prime}\right): x^{\prime} \in X(x)\right\}, \quad B^{\prime}=\left\{\left(y, y^{\prime}\right): y^{\prime} \in Y(y)\right\} .
$$

From the definition, $\left(x, x^{\prime}\right)+\left(y, y^{\prime}\right) \in \mathcal{A} \Longleftrightarrow x^{\prime}+y^{\prime}=(x+y)^{2}$. Thus $S=\left|\left\{\left(b, b^{\prime}\right) \in B \times B^{\prime}: b+b^{\prime} \in \mathcal{A}\right\}\right|$ and we apply Theorem 2.1,

Corollary 4.1. Let $A_{1}, A_{2}, A_{3}, A_{4} \subset \mathbb{F}_{q}$. Then, the number of solutions of the equation

$$
x_{1}+x_{2}=\left(x_{3}+x_{4}\right)^{2}, \quad x_{i} \in A_{i}
$$

is

$$
S=\frac{\left|A_{1}\right|\left|A_{2}\right|\left|A_{3}\right|\left|A_{4}\right|}{q}+\theta \sqrt{q\left|A_{1}\right|\left|A_{2}\right|\left|A_{3}\right|\left|A_{4}\right|}, \quad|\theta| \leq 1 .
$$

In particular, the number of solutions of

$$
x_{1}+x_{2}=z^{2}, \quad x_{1} \in A_{1}, x_{2} \in A_{2}, z \in \mathbb{F}_{q}
$$


is

$$
\left|A_{1}\right|\left|A_{2}\right|+\theta \sqrt{\left|A_{1}\right|\left|A_{2}\right| q}
$$

for some $\theta$ with $|\theta| \leq 1$.

Proof. The first part of the statement follows from Theorem 4.1 by taking

$$
X(x)=\left\{\begin{array}{l}
A_{1}, \quad x \in A_{3} \\
\emptyset \quad \text { otherwise }
\end{array} \quad \text { and } \quad Y(x)= \begin{cases}A_{2}, & x \in A_{4} \\
\emptyset & \text { otherwise }\end{cases}\right.
$$

The second part of the statement follows from the fact that if $A_{3}=A_{4}=\mathbb{F}_{q}$ then each solution of (4.2) corresponds to exactly $q$ solutions of (4.1).

Shkredov [13] used Weil's bound for exponential sums with multiplicative characters to prove the following result for $q=p$ prime and the condition $\left|X_{1}\right|\left|X_{2}\right|>20 p$.

Corollary 4.2. Let $X_{1}, X_{2} \subset \mathbb{F}_{q},\left|X_{1}\right|\left|X_{2}\right|>2 q$. Then there exist $x, y \in \mathbb{F}_{q}$ such that $x+y \in X_{1}$ and $x y \in X_{2}$.

Proof. The number of such that pairs $(x, y)$ is equal to the number of solutions of the equation

$$
\left(x_{1} / 2-z\right)\left(x_{1} / 2+z\right)=x_{2}, \quad x_{1} \in X_{1}, x_{2} \in X_{2}, z \in \mathbb{F}_{q} .
$$

We observe that this equation is equivalent to the equation $\left(x_{1} / 2\right)^{2}-x_{2}=z^{2}$. In order to apply (4.2) we split $X_{1}=X_{11} \cup X_{12}$ in such a way that the squares in each set are all distinct. Then we apply (4.1) separately to $A_{1}=\left\{x_{1}^{2} / 2: x_{i} \in\right.$ $\left.X_{11}\right\}, A_{2}=-X_{2}$ and to $A_{1}=\left\{x_{1}^{2} / 2: x_{i} \in X_{12}\right\}, A_{2}=-X_{2}$. It follows that the number of solutions of the equation $\left(x_{1} / 2\right)^{2}-x_{2}=z^{2}, \quad x_{1} \in X_{1}, x_{2} \in X_{2}, z \in \mathbb{F}_{q}$ is positive in view of

$$
\left|X_{11}\right|\left|X_{2}\right|-\sqrt{\left|X_{11}\right|\left|X_{2}\right| q}+\left|X_{12}\right|\left|X_{2}\right|-\sqrt{\left|X_{12}\right|\left|X_{2}\right| q} \geq\left|X_{1}\right|\left|X_{2}\right|-\sqrt{2\left|X_{1}\right|\left|X_{2}\right| q}>0 .
$$

Sárközy [11, 12] using exponential sums, obtained asymptotic formula for the number of solutions of the congruences $x_{1} x_{2}-x_{3} x_{4} \equiv \lambda(\bmod p)$ and $x_{1} x_{2}-x_{3}-$ $x_{4} \equiv \lambda(\bmod p), x_{i} \in X_{i}$. In [7] these results have been proved in any finite fields. We derive Sarkőzy's results directly from our Theorem 2.1.

Theorem 4.2. For $x \in \mathbb{F}_{q}^{*}, y \in \mathbb{F}_{q}^{*}$ let $X(x), Y(y)$ be any subsets of $\mathbb{F}_{q}$. Then for the number $S$ of solutions of the equation

$$
x^{\prime}+y^{\prime}=x y, \quad x^{\prime} \in X(x), y^{\prime} \in Y(y)
$$

we have

$$
S=\frac{T}{q}+\theta \sqrt{q T}, \quad|\theta| \leq 1+o(1)
$$


where $T=\left(\sum_{x}|X(x)|\right)\left(\sum_{x}|Y(x)|\right)$.

Proof. We consider the Sidon set $\mathcal{A}=\left\{\left(x, g^{x}\right): x \in \mathbb{Z}_{q-1}\right\}$ and the sets

$$
B=\left\{\left(\log x, x^{\prime}\right): x^{\prime} \in X(x)\right\}, \quad B^{\prime}=\left\{\left(\log y, y^{\prime}\right): y^{\prime} \in Y(y)\right\} .
$$

We observe that

$$
\left(\log x, x^{\prime}\right)+\left(\log y, y^{\prime}\right) \in \mathcal{A} \Longleftrightarrow x^{\prime}+y^{\prime}=g^{\log x+\log y}=x y .
$$

Thus $S=\left|\left\{\left(b, b^{\prime}\right) \in B \times B^{\prime}: b+b^{\prime} \in \mathcal{A}\right\}\right|$ and then we apply Theorem 2.1.

Corollary 4.3. Let $X_{1}, X_{2} \subset \mathbb{F}_{q}^{*}$ and $X_{3}, X_{4} \subset \mathbb{F}_{q}$. The number $S$ of solutions of the equation

$$
x_{1} x_{2}=x_{3}+x_{4}, \quad x_{i} \in X_{i}
$$

is

$$
S=\frac{\left|X_{1}\right|\left|X_{2}\right|\left|X_{3}\right|\left|X_{4}\right|}{q}+\theta \sqrt{\left|X_{1}\right|\left|X_{2}\right|\left|X_{3}\right|\left|X_{4}\right| q}, \quad|\theta| \leq 1+o(1)
$$

Proof. We take $X(x)$ and $Y(y)$ as in Corollary 4.1 and use Theorem 4.2.

Corollary 4.4. Let $X_{1}, X_{2} \subset \mathbb{F}_{q}^{*}$ and $X_{3}, X_{4} \subset \mathbb{F}_{q}$. The number $S$ of solutions of the equation

$$
x_{2} x_{3}-x_{1} x_{4}=1, \quad x_{i} \in X_{i}
$$

is

$$
S=\frac{\left|X_{1}\right|\left|X_{2}\right|\left|X_{3}\right|\left|X_{4}\right|}{q}+\theta \sqrt{\left|X_{1}\right|\left|X_{2}\right|\left|X_{3}\right|\left|X_{4}\right| q}, \quad|\theta| \leq 1+o(1)
$$

Proof. In Theorem 4.2 we take

$$
X(x)=\left\{\begin{array}{l}
x X_{3}, x \in X_{1}^{-1} \\
\emptyset \quad \text { otherwise }
\end{array} \quad \text { and } \quad Y(y)=\left\{\begin{array}{l}
-y X_{4}, y \in X_{2}^{-1} \\
\emptyset \text { otherwise }
\end{array} .\right.\right.
$$

In this way we arrive at the equation $x_{1}^{-1} x_{2}^{-1}=x_{1}^{-1} x_{3}-x_{2}^{-1} x_{4}$, which is equivalent to the equation of the corollary.

Theorem 4.3. For $x \in \mathbb{F}_{q}^{*}, y \in \mathbb{F}_{q}^{*}$, let $X(x), Y(y)$ be any subsets of $\mathbb{F}_{q}^{*}$. The number $S$ of solutions of the equation

$$
x y-x^{\prime} y^{\prime}=1, \quad x, y \in \mathbb{F}_{q}^{*}, x^{\prime} \in X(x), y^{\prime} \in Y(y),
$$

is

$$
S=\frac{T}{q}+\theta \sqrt{T q}, \quad|\theta| \leq 1+o(1),
$$

where $T=\left(\sum_{x}|X(x)|\right)\left(\sum_{x}|Y(x)|\right)$. 
Proof. We consider the Sidon set $\mathcal{A}=\left\{(x, y): g^{x}-g^{y}=1\right\} \subset \mathbb{Z}_{q-1} \times \mathbb{Z}_{q-1}$ and the sets $B=\left\{\left(\log x, \log x^{\prime}\right): x^{\prime} \in X(x)\right\}$ and $B^{\prime}=\left\{\left(\log y, \log y^{\prime}\right): y^{\prime} \in Y(y)\right\}$. It is clear that $S=\left|\left\{\left(b, b^{\prime}\right) \in B \times B^{\prime}: b+b^{\prime} \in \mathcal{A}\right\}\right|$. Now we apply Theorem 2.1 .

We observe that this theorem also gives an alternative proof of Corollary 4.4 by taking $X(x)$ and $Y(y)$ as in Corollary 4.1 .

\section{Distribution of Sidon Sets And applications}

Let $\mathcal{A}$ be a Sidon set in $G$. For any set $B \subset G$ we write $E_{\mathcal{A}}(B)=|\mathcal{A} \cap B|-\frac{|B||\mathcal{A}|}{|G|}$.

The following lemma and Lemma 3.1 will be the main tools to prove asymptotic estimates for $|\mathcal{A} \cap B|$ in a class of problems. For simplicity we restrict ourselves to the cases when $\mathcal{A}$ is one of the three Sidon sets described in Section 2.

Lemma 5.1. Let $\mathcal{A}$ be one of the three Sidon sets described in section $\S 2$ and $B \subset G$. For any set $C \subset G$, there exists $c \in C$ such that

$$
\left|E_{\mathcal{A}}(B)\right| \leq 2\left(q \frac{|B|}{|C|}\right)^{1 / 2}+\left|E_{\mathcal{A}}\left(B^{c}\right)\right|+\left|E_{\mathcal{A}}\left(B_{c}\right)\right|
$$

where $B^{c}=B \backslash(B+c)$ and $B_{c}=(B+c) \backslash B$.

Proof. We have

$$
\begin{aligned}
E_{\mathcal{A}}(B)=|\mathcal{A} \cap B|-\frac{|\mathcal{A}||B|}{|G|} & =\frac{1}{|C|} \sum_{c \in C}\left(|\mathcal{A} \cap(B+c)|-\frac{|\mathcal{A}||B|}{|G|}\right) \\
& +\frac{1}{|C|} \sum_{c \in C}(|\mathcal{A} \cap B|-|\mathcal{A} \cap(B+c)|) .
\end{aligned}
$$

We observe that

$$
\sum_{c \in C}\left(|\mathcal{A} \cap(B+c)|-\frac{|\mathcal{A}||B|}{|G|}\right)=|\{(b, c) \in B \times C: b+c \in \mathcal{A}\}|-\frac{|\mathcal{A}||B||C|}{|G|}
$$

Hence, by Theorem 2.1, the absolute value of this sum is bounded by $2(q|B||C|)^{1 / 2}$.

Since $\left|B_{c}\right|=\left|B^{c}\right|$, for the second sum in (5.1) we have

$$
\begin{aligned}
|\mathcal{A} \cap B|-|\mathcal{A} \cap(B+c)| & =\left|\mathcal{A} \cap B_{c}\right|-\left|\mathcal{A} \cap B^{c}\right| \\
& =\left(\left|\mathcal{A} \cap B_{c}\right|-\frac{|\mathcal{A}|\left|B_{c}\right|}{|G|}\right)-\left(\left|\mathcal{A} \cap B^{c}\right|-\frac{|\mathcal{A}|\left|B^{c}\right|}{|G|}\right) \\
& =E_{\mathcal{A}}\left(B_{c}\right)-E_{\mathcal{A}}\left(B^{c}\right) .
\end{aligned}
$$


Thus

$$
\left|\frac{1}{|C|} \sum_{c \in C}(|\mathcal{A} \cap B|-|\mathcal{A} \cap(B+c)|)\right| \leq \max _{c \in C}\left(\left|E_{\mathcal{A}}\left(B_{c}\right)\right|+\left|E_{\mathcal{A}}\left(B^{c}\right)\right|\right) .
$$

In the special case when $B$ is a subgroup we can take $C=B$ and then $B^{c}=$ $B_{c}=\emptyset$ for any $c \in C$. Thus, in this case we have

$$
\left|E_{\mathcal{A}}(B)\right| \ll q^{1 / 2} \text {. }
$$

As a corollary we obtain a well known result on the Fermat equation in finite fields.

Corollary 5.1. Let $Q, Q^{\prime}$ be subgroups of $\mathbb{F}_{q}^{*}$. We have

$$
\left|\left\{(x, y) \in Q \times Q^{\prime}: x+y=1\right\}\right|=\frac{|Q|\left|Q^{\prime}\right|}{q}+O(\sqrt{q}) \text {. }
$$

In particular, if $p \gg(r s)^{2}$ the Fermat congruence $x^{r}+y^{s} \equiv 1(\bmod p)$ has nontrivial solutions.

Proof. Consider the Sidon set $\mathcal{A}=\left\{(x, y): g^{x}+g^{y}=1\right\}$ and take $B=C=$ $Q \times Q^{\prime}$

In applications, the strategy is to take a large set $C$ such that $\left|B^{c}\right|$ and $\left|B_{c}\right|$ are small compared with $|B|$. This is possible when $B$ has some specific regularity properties (subgroups, cartesian product of arithmetic progressions, convex sets, etc.) We illustrate our method with an example.

Theorem 5.1. Let $I, J \subset \mathbb{Z}_{p-1}$ be intervals. For any positive integer $r$ we have

$$
\left\{(x, y) \in I \times J: g^{x}-g^{y} \equiv \lambda \quad(\bmod p)\right\}=\frac{|I||J|}{p}+4^{r} \theta\left(\left(\frac{|I||J|}{p^{3 / 2}}\right)^{1 / r}+1\right) \sqrt{p},
$$

with $|\theta| \leq 1$.

Proof. We proceed by induction on $r$. We consider the Sidon set $\mathcal{A}=\{(x, y)$ : $\left.g^{x}-g^{y}=\lambda\right\} \subset \mathbb{Z}_{p-1} \times \mathbb{Z}_{p-1}$ and the set $B=I \times J$. Then, applying Lemma 3.1, we get

$$
|\mathcal{A} \cap B| \leq \frac{|B+B|}{p}+2 \sqrt{p \frac{|B+B|}{|B|}} \leq \frac{4|I||J|}{p}+4 \sqrt{p} .
$$

Since $\left|E_{\mathcal{A}}(B)\right| \leq \max \left(\frac{|B||\mathcal{A}|}{|G|},|\mathcal{A} \cap B|\right)$, we have that $\left|E_{\mathcal{A}}(B)\right| \leq \frac{4|I||J|}{p}+4 \sqrt{p}$, which proves Theorem 5.1 for $r=1$. Now we assume that Theorem 5.1 is true for some $r$ and we proved it for $r+1$.

We consider the auxiliar set $C=I^{\prime} \times J^{\prime}$ where $I^{\prime}=\{0, \ldots,\lfloor\alpha|I|\rfloor\}$ and $J^{\prime}=$ $\{0, \ldots,\lfloor\alpha|J|\rfloor\}$ for a suitable $\alpha$. We observe that $|C| \geq \alpha^{2}|I||J|$. Lemma 5.1 
gives $\left|E_{\mathcal{A}}(B)\right| \leq 2 \frac{p^{1 / 2}}{\alpha}+\left|E_{\mathcal{A}}\left(B^{c}\right)\right|+\left|E_{\mathcal{A}}\left(B_{c}\right)\right|$. Now we observe that $B+c$ is a small translation of the rectangle $B=I \times J$. Thus we can write $B^{c}=B_{1} \cup B_{2}$ and $B_{c}=B_{3} \cup B_{4}$ where the sets $B_{i}$ are rectangles with $\left|B_{i}\right| \leq \alpha|I||J|$.

Thus, $\left|E_{\mathcal{A}}(B)\right| \leq 2 \frac{p^{1 / 2}}{\alpha}+\left|E_{\mathcal{A}}\left(B_{1}\right)\right|+\left|E_{\mathcal{A}}\left(B_{2}\right)\right|+\left|E_{\mathcal{A}}\left(B_{3}\right)\right|+\left|E_{\mathcal{A}}\left(B_{4}\right)\right|$.

Taking into account the induction hypothesis for each $B_{i}$ we have

$$
\left|E_{\mathcal{A}}(B)\right| \leq 2 \frac{p^{1 / 2}}{\alpha}+4 \theta_{r}\left(\left(\frac{\alpha|I||J|}{p^{3 / 2}}\right)^{1 / r}+1\right) \sqrt{p} .
$$

Taking $\alpha=\frac{1}{4^{r}}\left(\frac{p^{3 / 2}}{|I||J|}\right)^{1 /(r+1)}$ we obtain the required estimate.

It should be mentioned that, for the particular case $|I|=|J|$, Garaev obtained the error term $O\left(|I|^{2 / 3} \log ^{2 / 3}\left(|I| p^{-3 / 4}+2\right)+p^{1 / 2}\right)$. We note that the error term in Theorem 5.1 is smaller than Garaev's error term. Furthermore, in the range $p^{3 / 2} \ll|I||J| \ll p^{3 / 2}(\log p)^{\log \log p}$ our error term is smaller than the error term $O\left(p^{1 / 2} \log ^{2} p\right)$ established in [10]. For arbitrary intervals, Theorem 5.1] gives $O_{\epsilon}\left(p^{1 / 2+\epsilon}\right)$ for any $\epsilon>0$, which is only slightly weaker than $O\left(p^{1 / 2} \log ^{2} p\right)$.

Finally, we remark that the analogy of Theorem 5.1 also holds for some other problems of similar flavor, like estimating $\left\{x \in I: x^{2} \in J\right\}$ or $\left\{x \in I: g^{x} \in J\right\}$. These are achieved by employing suitable Sidon sets.

Acknowledgments: Parts of this work have been presented in several places: Conference on Arithmetic Combinatorics (IAS Princeton 2007), Primera Reunión Conjunta Sociedad Matemática Mexicana-Real Sociedad Matemática Española (Oaxaca, México, 2009) and Discrete Analysis Seminar (University of Cambridge, 2010). I would like to thank these institutions for their hospitality. Also I want to thank Igor Shparlinsky and M. Garaev for useful conversations about this work.

\section{REFERENCES}

[1] P. Csikvári, K. Gyarmati and A. Sárközy, Density and Ramsey type results on algebraic equations with restricted solution sets, Combinatorica, to appear.

[2] M. Z. Garaev, The sum-product estimate for large subsets of prime fields. Proc. Amer. Math. Soc. 136 (2008) n.8, 2735-2739.

[3] M. Z. Garaev, On the logarithmic factor in error term estimates in certain additive congruence problems, Acta Arithmetica 124 (2006) n.1, 33-40.

[4] M. Z. Garaev and Ka-Lam Kueh, Distribution of special sequences modulo a large prime, International Journal of Mathematics and Mathematical Sciences, 50 (2003) n.50, 31893194.

[5] M. Z. Garaev and C. Shen, On the size of the set $A(A+1)$, Mathematische Zeitschrift, 265 (2010) n.1, 125-132.

[6] V. C. García, A note on an additive problem with powers of a primitive root. Bol. Soc. Mat. Mexicana (3) 11 (2005) n.1, 1-4. 
[7] K. Gyarmati and A. Sárközy, Equations in finite fields with restricted solution sets, II. (Algebraic equations.), Acta Math. Hungar. 119 (2008) n.3, 259-280.

[8] Derrick Hart, Liangpan Li, Chun-Yen Shen, Fourier analysis and expanding phenomena in finite fields. arXiv:0909.5471

[9] S. V. Konyagin, Bounds of exponential sums over subgroups and Gauss sums, Proc 4th Intern. Conf. Modern Problems of Number Theory and Its Applications, Moscow Lomonosov State Univ., Moscow, (2002) 86-114 (in Russian)

[10] Z. Rudnik and A. Zaharescu, The distribution of spacing between small powers of a primitive root, Israel Journal of Mathematics 120 (2000), 271-287.

[11] A. Sárközy, On sums and products on residues modulo p. Acta Arithmetica 118 (2005), n.4, 403-409.

[12] A. Sárközy, On products and shifted products of residues modulo $p$. Proceedings of CANT 2005. Integers $\mathbf{8}(2),(2008)$

[13] I. D. Shkredov, On monochromatic solutions of some nonlinear equations in $\mathbb{Z} / \mathbb{Z}_{p}$, arXiv:0909.3269

[14] J. Solymosi, Incidences and the spectra of graphs, Combinatorial Number Theory and Additive Group Theory, Advanced Courses in Mathematics - CRM Barcelona Birkhäuser Basel (2009), 299-314.

[15] Vinh. Szemeredi-Trotter type theorem and sum-product estimate in finite fields, arXiv:0711.4427v1 [math.CO]

Instituto de Ciencias Matemáticas (CSIC-UAM-UC3M-UCM) and Departamento de Matemáticas, Universidad Autónoma de Madrid, 28049, Madrid, España

E-mail address: franciscojavier.cilleruelo@uam.es 